\title{
Susceptibilidad antimicrobiana en Chile 2012
}

\author{
Marcela Cifuentes-D., Francisco Silva, Patricia García, Helia Bello, Isabel Briceño, Mario Calvo-A. y \\ Jaime Labarca, en representación del Grupo Colaborativo de Resistencia Bacteriana de Chile*
}

\section{Antimicrobial susceptibility in Chile 2012}

Bacteria antimicrobial resistance is an uncontrolled public health problem that progressively increases its magnitude and complexity. The Grupo Colaborativo de Resistencia, formed by a join of experts that represent 39 Chilean health institutions has been concerned with bacteria antimicrobial susceptibility in our country since 2008 . In this document we present in vitro bacterial susceptibility accumulated during year 2012 belonging to 28 national health institutions that represent about $36 \%$ of hospital discharges in Chile. We consider of major importance to report periodically bacteria susceptibility so to keep the medical community updated to achieve target the empirical antimicrobial therapies and the control measures and prevention of the dissemination of multiresistant strains.

Key words: Drug resistance, antimicrobial agents, epidemiology, antibiotics, bacterial resistance, multi-resistant, pan-drug resistant, antimicrobial susceptibility, antimicrobial resistante, surveillance networks.

Palabras clave: Resistencia, antimicrobianos, antimicrobianos, epidemiología, susceptibilidad, red de vigilancia, multiresistentes, panresistentes, susceptibilidad antimicrobiana, resistencia antimicrobiana, red de vigilancia.

\section{Introducción}

$\mathrm{E}$ 1 Grupo Colaborativo de Resistencia Bacteriana (GCRB) fue constituido el año 2007, incorporándose posteriormente como un grupo de trabajo a la Sociedad Chilena de Infectología. En la actualidad cuenta con la representación de 39 centros de salud, los cuales aportan información sobre la susceptibilidad y/o resistencia de las bacterias a los agentes antibacterianos así como también del consumo de éstos. Este grupo denominado "Grupo Colaborativo de Resistencia Bacteriana (GCRB), Chile” publicó el año 2011 el primer reporte de susceptibilidad antimicrobiana ${ }^{1}$. En aquella oportunidad, se reportó la susceptibilidad del año 2009 e incluyó a 12 hospitales del país, con énfasis en la información y las limitaciones de la misma.

Actualmente, la resistencia bacteriana es considerada un problema prioritario de salud pública². Lamentable- mente, lejos de solucionarse, este problema continúa aumentando, tanto por su incidencia, como debido a la variedad de mecanismos de resistencia descritos. Esto último se ve favorecido por la presencia de clones de alta transmisibilidad ${ }^{3}$ y elementos genéticos móviles, que facilitan la diseminación de genes de resistencia entre las bacterias $^{4-6}$. Tanta es la importancia de este problema, que en el año 2011 la OMS lo consideró como tema prioritario en el Día Mundial de la Salud?

En la actualidad, muchas especies bacterianas presentan fenotipos de multi-resistencia, incluyendo nuevos mecanismos de resistencia ${ }^{8,9}$. Para estas bacterias pocos antimicrobianos persisten activos, entre ellos carbapenémicos, colistín y tigeciclina para los bacilos gramnegativos y daptomicina y linezolid, en el caso de cocáceas grampositivas ${ }^{10-12}$.

De esta manera, es crítico contar con una información continuamente actualizada en el tiempo respecto de
Hospital Clínico San Borja Arriarán, Santiago (MC-D). Hospital Clínico Universidad de Chile, Santiago (FS). Pontificia Universidad Católica de Chile, Santiago. Escuela de Medicina, Departamento de Laboratorios Clínicos (PG). Departamento de Enfermedades Infecciosas (JL). Universidad de Concepción, Concepción. Facultad de Ciencias Biológicas (HB). Hospital Naval "Almirante Nef", Viña del Mar (IB). Hospital Regional de Valdivia, Valdivia (MC-A).

Conflictos de interés: Los autores declaran que han recibido apoyo logístico de MSD Chile, BD Chile y Pfizer Inc. Chile para la organización de las reuniones de coordinación del grupo colaborativo. Ninguna de las empresas ha tenido influencia sobre las decisiones del método de vigilancia, selección de los participantes del grupo, generación o análisis de los resultados. Los autores no han recibido remuneración alguna de ellos ni de otra institución por su participación en el grupo colaborativo.

Recibido: 28 de febrero de 2014 Aceptado: 21 de marzo de 2014

Correspondencia a: Jaime Labarca Labarca jlabarca@med.puc.cl

\footnotetext{
*Grupo Colaborativo de Resistencia Bacteriana de Chile**: Hospital Leonardo Guzmán, Antofagasta (Eva Mejías). Hospital Gustavo Fricke, Viña del Mar (Alejandro Joyas). Hospital Dr. Carlos Van Buren, Valparaíso. (Gerardo Peralta, Gonzalo Wilson). Hospital Naval Almirante Nef, Viña del Mar (Isabel Briceño). Hospital Dr. Eduardo Pereira, Valparaíso (Daniel Guzmán, Rubén Muñoz). Hospital Higueras, Talcahuano (Fabiola Salgado, Álvaro Llancaqueo). Hospital Clínico Regional de Concepción Dr. Guillermo Grant Benavente, Concepción (Henriette Chabouty, Gisela Riedel). Facultad de Ciencias Biológicas, Universidad de Concepción (Gerardo González, Helia Bello, Mariana Domínguez). Hospital Regional de Temuco Dr. Hernán Henríquez (Vijna Illesca). Hospital Clínico Regional de Valdivia, Valdivia (M. Carolina Cruz). Universidad Austral de Chile, Valdivia (Mario Calvo). Hospital Puerto Montt, Puerto Montt (M. Luisa Rioseco). Hospital de la Fuerza Aérea Dr. Raúl Yazigi J., Santiago (Erna Cona, Michel Serri). Hospital DIPRECA, Santiago (Margareta Muhlhauser). Hospital Militar de Santiago, Santiago (Stephanie Braun, Lorena Porte). Hospital Clínico Universidad de Chile, Santiago (Francisco Silva, M. Eugenia Pinto). Departamento de Laboratorios Clínicos, Escuela de Medicina, Pontificia Universidad Católica de Chile, Santiago, Santiago (Patricia García). Hospital Clínico Pontificia Universidad Católica de Chile, Santiago (Jaime Labarca). Hospital Dr. Exequiel González Cortés, Santiago (Carmen Mendoza). Hospital de Niños Dr. Luis Calvo Mackenna, Santiago (Isabel Álvarez). Hospital Clínico de Niños Roberto del Río, Santiago (Dona Benadof). Hospital Barros Luco Trudeau, Santiago (Sofía Palma). Hospital Del Salvador, Santiago (Chrystal Juliet, Alejandra Fernández). Hospital Clínico San Borja Arriarán, Santiago (Marcela Cifuentes). Hospital Padre Hurtado, Santiago (Pamela Rojas). Hospital San Juan de Dios, Santiago (Alejandra Céspedes, José M. Arancibia). Hospital Dr. Sótero del Río, Santiago (Pablo Aguilera). Integramédica Laboratorio Clínico, Santiago (Rossanna Camponovo). Clínica Alemana, Santiago (Lorena Porte). Clínica Las Condes, Santiago (Beatrice Hervé). Clínica Dávila, Santiago (Cecilia Tapia). Revista Chilena de Infectología, Editor (José Cofré)

**Enumeración según orden geográfico de las instituciones (de norte a sur del país)
} 
la susceptibilidad in vitro que exhiben nuestras cepas bacterianas a los agentes antimicrobianos, para evaluar las tendencias, establecer tratamientos empíricos fundamentados y medidas epidemiológicas de control, locales y nacionales ${ }^{13}$.

En el presente artículo, reportamos la susceptibilidad in vitro acumulada del año 2012 de 28 hospitales complejos de Chile con un número significativo de aislados clínicos.

\section{Materiales y Métodos}

Se solicitó la información sobre datos de susceptibilidad in vitro del año 2012 a 39 establecimientos de salud de la red pública y privada pertenecientes al GCRB, Chile. Se utilizó un sistema de recopilación de datos similar al previamente publicado por este grupo, de acuerdo a las sugerencias del Clinical and Laboratory Standards Institute (CLSI), generándose un consolidado de los mismos en una única base de datos global ${ }^{1,14,15}$.

La información correspondió sólo a muestras de origen clínico. Se solicitó un aislado por especie y por paciente. Se segregó la información por grupo etario (pediátricos $<15$ años y adulto $\geq 15$ años), por tipo de servicio clínico: unidad de paciente crítico (UPC), unidades de pacientes de baja complejidad (no UPC) y hospitalizados (para

Tabla 1. Especies bacterianas vigiladas según tipo de muestra y procedencia

\begin{tabular}{|c|c|c|}
\hline Microorganismo & Tipo de muestra & Procedencia de la muestra \\
\hline \multirow[t]{3}{*}{ Escherichia coli } & Orina (urocultivos) & Ambulatoria \\
\hline & & Hospitalizados \\
\hline & Sangre (hemocultivos) & Independiente de la procedencia \\
\hline \multirow[t]{3}{*}{ Klebsiella pneumoniae } & Cualquier tipo & Unidades de paciente críticos (UPC) \\
\hline & & Servicios no crítico (no UPC) \\
\hline & & Hospitalizados en general \\
\hline \multirow[t]{3}{*}{ Enterobacter cloacae } & Cualquier tipo & Unidades de paciente crítico (UPC) \\
\hline & & Servicios no críticos (no UPC) \\
\hline & & Hospitalizados en general \\
\hline \multirow[t]{3}{*}{ Pseudomonas aeruginosa } & Cualquier tipo & Unidades de paciente crítico (UPC) \\
\hline & & Servicios no críticos (no UPC) \\
\hline & & Hospitalizados en general \\
\hline \multirow[t]{3}{*}{ Acinetobacter baumannii } & Cualquier tipo & Unidades de paciente crítico (UPC) \\
\hline & & Servicios no críticos (no UPC) \\
\hline & & Hospitalizados en general \\
\hline \multirow[t]{3}{*}{ Staphylococcus aureus } & Cualquier tipo & Unidades de paciente crítico (UPC) \\
\hline & & Servicios no críticos (no UPC) \\
\hline & & Hospitalizados en general \\
\hline \multirow[t]{3}{*}{ Enterococcus faecium } & Cualquier tipo & Hospitalizados en general \\
\hline & & Unidades de paciente crítico (UPC) \\
\hline & & Servicios no críticos (no UPC) \\
\hline \multirow[t]{3}{*}{ Enterococcus faecalis } & Cualquier tipo & Hospitalizados en general \\
\hline & & Unidades de pacientes críticos \\
\hline & & Servicios no críticos \\
\hline
\end{tabular}

aquellos hospitales que no pueden diferenciar en UPC y no UPC). Sólo en el caso de Escherichia coli, se separó por tipo de muestra incluyendo hemocultivos, urocultivos ambulatorios y urocultivos hospitalizados por separado.

La información se expresó en porcentajes de susceptibilidad in vitro de acuerdo a las recomendaciones internacionales ${ }^{14}$. No hubo intervención alguna para modificar los procedimientos locales de toma de muestra, identificación, ni estudio de susceptibilidad in vitro por lo que cada hospital siguió los procedimientos internos respectivos.

En Chile, el Programa Nacional de Prevención y Control de Infecciones Asociadas a la Atención de Salud se encuentra regido actualmente por la Norma Técnica 124 de octubre de 2011. Dicha norma indica en su $5^{\circ}$ ámbito que los establecimientos de salud deben contar con un Laboratorio de Microbiología que provea información sobre la susceptibilidad antimicrobiana in vitro periódicamente, en forma rutinaria, por lo que no se consideró necesario someter a comité de ética este trabajo ${ }^{16}$. Sin embargo, cada laboratorio contó con la aprobación de su autoridad local o respectiva para el envío de sus datos, los que se manejaron de manera confidencial por parte del equipo coordinador que consolidó la información.

Los microorganismos seleccionados para el presente reporte fueron aquellos definidos en forma consensuada por el GCRB en sus reuniones periódicas y que correspondieron a: Staphylococcus aureus, Enterococcus faecalis, Enterococcus faecium, Escherichia coli, Klebsiella pneumoniae, Enterobacter cloacae, Pseudomonas aeruginosa y Acinetobacter baumannii (Tabla 1).

\section{Resultados}

Centros participantes: del total de 39 centros que participan en el GCRB, 28 centros enviaron sus datos para el período 2012 (Tabla 2). Estos centros representan un total de 12.115 camas de 15 del total de 29 Servicios de Salud del país, siendo al menos 1.341 de ellas, camas de pacientes críticos. Los centros se distribuyeron en las regiones de Antofagasta, Valparaíso, Metropolitana, BíoBío, La Araucanía, de Los Ríos y de Los Lagos (Figura 1).

De los 28 establecimientos, 15 correspondían a hospitales públicos generales, de alta o mediana complejidad, cuatro a hospitales institucionales (de las FFAA), tres a hospitales pediátricos, tres eran hospitales privados (clínicas), dos a hospitales universitarios y uno era un laboratorio de red de centros de atención abierta (Figura 1 y Tabla 2).

De los 28 centros, 27 son centros de atención cerrada y totalizaron 594.576 egresos para el año 2011, lo que corresponde a $36 \%$ de los egresos de ese año en el país.

Entre los centros de atención cerrada, la mayor parte $(25 / 28)$, informó sus cepas diferenciándolas en UPC y no $U P C$ y el resto informó la sumatoria de sus cepas de 
Tabla 2. Centros informantes de datos de susceptibilidad in vitro para el año 2012

\section{Centros Informantes 2012}

Hospital Regional de Antofagasta

Hospital Gustavo Fricke de Viña del Mar

Hospital Dr. Carlos Van Buren de Vaparaíso

Hospital Dr. Eduardo Pereira de Valparaíso

Hospital Naval "Almirante Nef", Valparaíso

Hospital Higueras de Talcahuano

Hospital Regional de Concepción, Dr. Guillermo Grant Benavente

Hospital Regional de Temuco, Dr. Hernán Henríquez

Hospital Regional de Valdivia

Hospital Regional de Puerto Montt

Hospital de la Fuerza Aérea "Dr. Raúl Yazigi"

Hospital DIPRECA

Hospital Militar de Santiago

Hospital Clínico Universidad de Chile

Hospital Clínico Pontificia Universidad Católica

Hospital Dr. Exequiel González Cortés

Hospital de Niños Dr. Luis Calvo Mackenna

Hospital de Niños Dr. Roberto del Río

Hospital Barros Luco Trudeau

Hospital Del Salvador

Hospital Padre Hurtado

Hospital Clínico San Borja Arriarán

Hospital San Juan de Dios

Hospital Dr. Sótero del Río

Integramédica

Clínica Alemana de Santiago

Clínica Dávila, Santiago

Clínica Las Condes, Santiago

hospitalizados por no ser posible obtener la información en forma separada de sus sistemas informáticos.

\section{Resultados acumulados de susceptibilidad in vitro}

\section{Staphylococcus aureus}

En las Tablas 3 y 4 se muestran los datos de susceptibilidad in vitro de $S$. aureus en adultos y niños, respectivamente. Destaca, en las cepas aisladas de adultos, la susceptibilidad in vitro a oxacilina de $51,7 \%$ (rango $14-89 \%$, datos no mostrados) v/s la de pediatría que es de $84,9 \%$. Por otro lado, la susceptibilidad in vitro a rifampicina y cotrimoxazol se mantiene en cifras cercanas a $97 \%$. No se detectó la presencia de cepas resistentes a vancomicina, ya fuese en niños o adultos.

\section{Enterococcus spp}

En la Tabla 5 se muestran los datos de susceptibilidad in vitro de Enterococcus spp. Puede observarse que

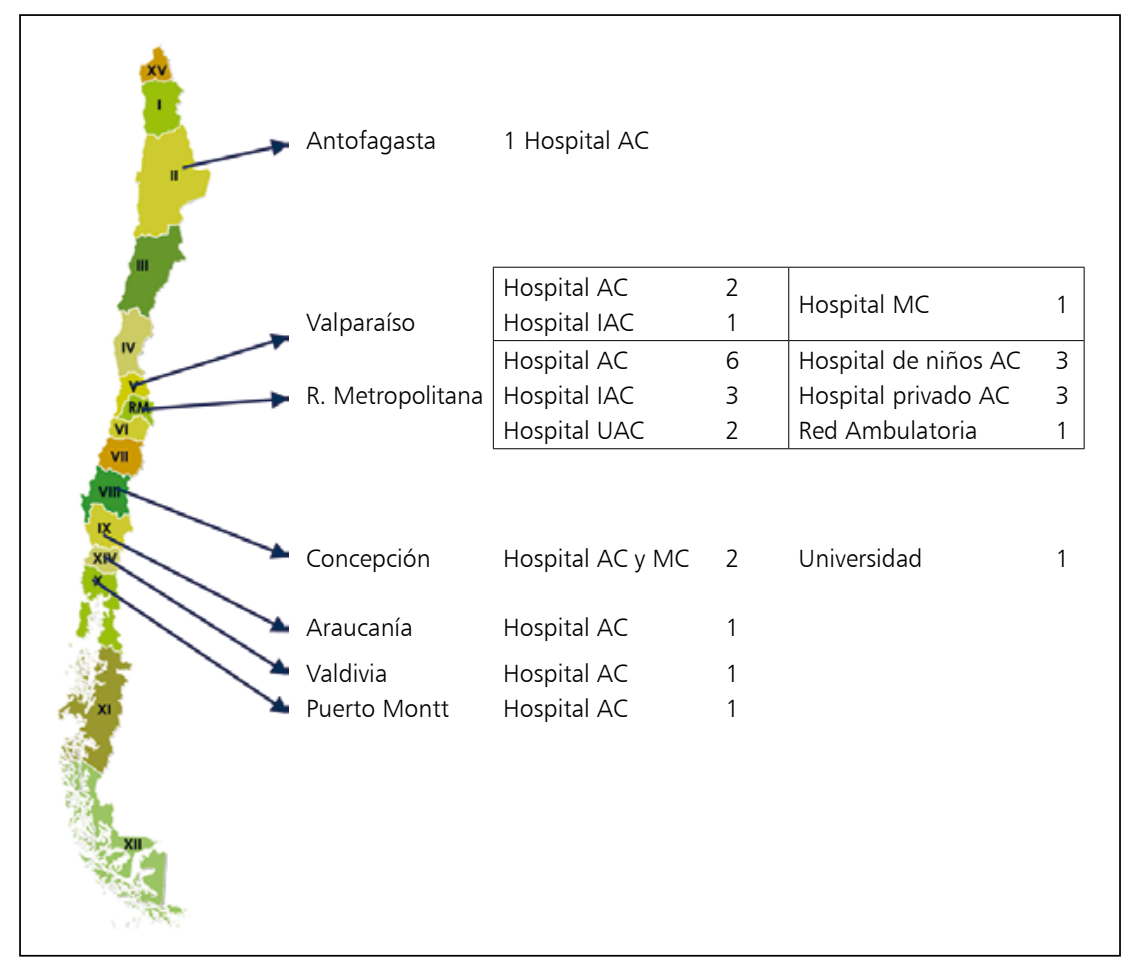

Figura 1. $A C=$ alta complejidad; $M C=$ mediana complejidad; $I A C=$ institucional alta complejidad; $\mathrm{UAC}=$ universitario alta complejidad.

Tabla 3. Susceptibilidad in vitro de Staphylococcus aureus aislados en el año 2012 en población adulta

\begin{tabular}{|lccc|} 
Antimicrobiano & UPC (N: 2.113) & No UPC (N: 4.382) & Hospitalizados (N: 7.055) \\
Cloxacilina & 41,7 & 53,7 & $\%$ \\
Vancomicina & 100,0 & 100,0 & 51,7 \\
Cotrimoxazol & 97,1 & 97,1 & 100,0 \\
Rifampicina & 96,0 & 98,5 & 97,2 \\
Eritromicina & 36,7 & 47,9 & 97,7 \\
Clindamicina & 39,7 & 50,2 & 45,9 \\
\hline Los datos están expresados en porcentaje de cepas susceptibles & 48,7 \\
\hline
\end{tabular}

Tabla 4. Susceptibilidad in vitro de Staphylococcus aureus aislados en el año 2012 en población pediátrica

\begin{tabular}{lccc|} 
Antimicrobiano & UPC (N: 265) & No UPC (N: 285) & Hospitalizados (N: 550) \\
\% & 86,1 & 83,8 & $\%$ \\
Cloxacilina & 100,0 & 100,0 & 84,9 \\
Vancomicina & 98,1 & 96,1 & 100,0 \\
Cotrimoxazol & 99,6 & 98,9 & 97,0 \\
Rifampicina & 78,5 & 78,9 & 99,3 \\
Eritromicina & 87,0 & 85,3 & 78,7 \\
Clindamicina & \multicolumn{2}{l}{} \\
\hline \multicolumn{2}{l}{ Los datos están expresados en porcentaje de cepas susceptibles. } \\
\hline
\end{tabular}


Tabla 5. Susceptibilidad in vitro de Enterococcus faecium y E. faecalis aislados en el año 2012 en población adulta

\begin{tabular}{lcccc} 
Antimicrobiano & \multicolumn{2}{c}{ E. faecium } & \multicolumn{2}{c}{ E. faecalis } \\
& $\begin{array}{c}\text { Hospitalizados } \\
\text { (N: } \mathbf{1 . 7 1 2}) \\
\%\end{array}$ & $\begin{array}{c}\text { UPC } \\
\text { (N: 557) } \\
\%\end{array}$ & $\begin{array}{c}\text { Hospitalizados } \\
\text { (N: } \mathbf{3 . 9 5 1 )}\end{array}$ & $\begin{array}{c}\text { No UPC } \\
\text { (N: 982) }\end{array}$ \\
Ampicilina & 9,2 & 6,5 & 95,2 & $\%$ \\
Vancomicina & 24,1 & 14,0 & 98,7 & 9,2 \\
Teicoplanina & $48,6^{*}$ & $29,5^{*}$ & $99,0^{*}$ & $58,2^{*}$
\end{tabular}

Los datos están expresados en porcentaje de cepas susceptibles. *Se cuenta con información de susceptibilidad in vitro a teicoplanina solamente en alrededor del $80 \%$ de las cepas totales.

Tabla 6. Susceptibilidad in vitro de Escherichia coli aisladas en el año 2012 en población adulta

\begin{tabular}{|c|c|c|c|}
\hline Antimicrobiano & $\begin{array}{c}\text { Urocultivos } \\
\text { ambulatorios } \\
\text { (N: 63.208) } \\
\%\end{array}$ & $\begin{array}{c}\text { Urocultivos } \\
\text { hospitalizados } \\
\text { (N: 10.887) } \\
\%\end{array}$ & $\begin{array}{l}\text { Hemocultivos } \\
\qquad \begin{array}{c}\text { (N: } 1.298) \\
\%\end{array}\end{array}$ \\
\hline Gentamicina & 91,9 & 86,1 & 83,1 \\
\hline Amikacina & 99,1 & 97,3 & 98,0 \\
\hline Ciprofloxacina & 77,2 & 69,7 & 67,7 \\
\hline Nitrofurantoína & 95,3 & 91,9 & - \\
\hline Cotrimoxazol & 71,7 & 67,5 & 63,8 \\
\hline Ampicilina & 43,7 & 37,0 & 36,9 \\
\hline Cefalotina & 62,4 & 55,7 & 58,6 \\
\hline Cefotaxima & 92,2 & 81,4 & 77,6 \\
\hline Piperacilina/tazobactam & 95,3 & 90,4 & 92,9 \\
\hline Ertapenem & 99,9 & 99,1 & 99,3 \\
\hline Imipenem & 99,6 & 98,4 & 99,9 \\
\hline Meropenem & 99,6 & 99,2 & 99,9 \\
\hline
\end{tabular}

Tabla 7. Susceptibilidad in vitro de Escherichia coli aisladas en el año 2012 en población pediátrica

\begin{tabular}{|c|c|c|c|}
\hline Antimicrobiano & $\begin{array}{c}\text { Urocultivos } \\
\text { ambulatorios } \\
\text { (N: } 2.948) \\
\%\end{array}$ & $\begin{array}{c}\text { Urocultivos } \\
\text { hospitalizados } \\
\text { (N: } 332 \text { ) } \\
\%\end{array}$ & $\begin{array}{l}\text { Hemocultivos } \\
\qquad \begin{array}{c}\text { (N: } 79) \\
\%\end{array}\end{array}$ \\
\hline Gentamicina & 90,4 & 89,9 & 85,7 \\
\hline Amikacina & 98,5 & 97,8 & 100,0 \\
\hline Ciprofloxacina & 92,1 & 90,1 & 88,6 \\
\hline Nitrofurantoína & 82,3 & 94,0 & - \\
\hline Cotrimoxazol & 70,3 & 70,6 & 64,8 \\
\hline Ampicilina & 35,6 & 31,9 & 30,4 \\
\hline Cefalotina & 58,8 & 48,3 & 40,0 \\
\hline Cefotaxima & 65,7 & 88,6 & 92,4 \\
\hline Ertapenem & 100,0 & 100,0 & 100,0 \\
\hline Imipenem & 100,0 & 100,0 & 100,0 \\
\hline Meropenem & 100,0 & 100,0 & 100,0 \\
\hline
\end{tabular}

Los datos están expresados en porcentaje de cepas susceptibles. existe una gran diferencia entre las especies E. faecalis y E. faecium aisladas de adultos; la susceptibilidad global a vancomicina es de 98,7 y $24 \%$, respectivamente. No se calculó la susceptibilidad in vitro de Enterococcus spp. en niños por el bajo número de aislados obtenidos.

\section{Escherichia coli}

La susceptibilidad in vitro de cepas de E. coli aisladas desde urocultivos de pacientes hospitalizados y ambulatorios, así como también de hemocultivos, se detalla para adultos (Tabla 6) y en niños (Tabla 7). La susceptibilidad in vitro a cefalosporinas de tercera generación $(\mathrm{C} 3 \mathrm{G})$ varía entre $92,2 \%$ en urocultivos ambulatorios, $81,4 \%$ en urocultivos de pacientes hospitalizados y $77,6 \%$ en hemocultivos. Se estima que la susceptibilidad in vitro de E. coli a $\mathrm{C} 3 \mathrm{G}$ podría acercarse a la prevalencia de $\beta$-lactamasas de espectro extendido (BLEE). A nitrofurantoína se mantiene una susceptibilidad in vitro de $95 \%$, siendo éste el antimicrobiano con mayor actividad, entre los fármacos orales, sobre cepas de E. coli provenientes de urocultivos ambulatorios. Para ciprofloxacina, cotrimoxazol y cefalosporinas de $1^{\mathrm{a}}$ generación la susceptibilidad contra dicho microorganismo no supera el $80 \%$.

\section{Klebsiella pneumoniae}

En las Tablas 8 y 9 se presenta la susceptibilidad in vitro de $K$. pneumoniae en adultos y niños, respectivamente. Destaca una baja susceptibilidad in vitro en cepas de adultos a C3G y a ertapenem, fluctuando entre $31,7-27 \%$ y 76,3-74,2\%, respectivamente. La actividad antibacteriana de los otros carbapenémicos se mantiene en cifras superiores a $94 \%$ de susceptibilidad. En el caso de piperacilina/tazobactam, la susceptibilidad in vitro no supera el $45 \%$.

\section{Enterobacter cloacae}

En la Tabla 10 se observa una susceptibilidad in vitro similar aunque ligeramente mejor a ertapenem que la encontrada en las cepas de $K$. pneumoniae. En adultos fluctúa en un rango de 79,3 a 82,6\%. La susceptibilidad a cefepime es similar a ertapenem, con un rango de 78,8 a $84,4 \%$. Del mismo modo, la susceptibilidad in vitro a carbapenémicos se observa discretamente mejor.

\section{Pseudomonas aeruginosa}

En las Tablas 11 (adultos) y 12 (niños), se puede observar la susceptibilidad de $P$. aeruginosa. Para ningún antimicrobiano, con excepción de colistín, se encontró una susceptibilidad in vitro superior a $80 \%$. En el caso de los carbapenémicos, la susceptibilidad in vitro promedio presentada por las cepas de $P$. aeruginosa es cercana a $65 \%$ para imipenem y $68 \%$ para meropenem manteniéndose susceptibilidad in vitro semejante a ciprofloxacina, cefepime, piperacilina/tazobactam y ceftazidima. Amikacina mostró la mejor actividad in vitro, en torno a $81 \%$, y colistín a $95 \%$. Las cepas pediátricas mostraron un comportamiento similar aunque discretamente mejor.

\section{Acinetobacter baumannii}

En las Tablas 13 (adultos) y 14 (niños) se observa que las cepas de $A$. baumannii presentaron $99 \%$ de susceptibilidad in vitro para colistín. La susceptibilidad in vitro observada a ampicilina/sulbactam fue $39 \%$ en adultos. Entre los aminoglucósidos la susceptibilidad in vitro a amikacina fue de $27 \%$ versus $48 \%$ a gentamicina. El promedio 
Tabla 8. Susceptibilidad in vitro de Klebsiella pneumoniae aisladas en el año 2012 en población adulta

\begin{tabular}{|lccc|} 
Antimicrobiano & $\begin{array}{c}\text { Hospitalizados } \\
\text { (N: } \mathbf{5 . 6 8 9}) \\
\text { \% }\end{array}$ & $\begin{array}{c}\text { UPC } \\
\text { (N: 1.746) } \\
\text { \% }\end{array}$ & $\begin{array}{c}\text { No UPC } \\
\text { (N: } \mathbf{3 . 3 5 2} \\
\text { \% }\end{array}$ \\
Ertapenem & 76,3 & 74,2 & 75,0 \\
Imipenem & 98,7 & 98,4 & 98,7 \\
Meropenem & 94,2 & 93,8 & 93,7 \\
Amikacina & 86,2 & 87,3 & 85,5 \\
Ciprofloxacina & 39,8 & 38,2 & 38,0 \\
Piperacilina/tazobactam & 44,6 & 39,7 & 42,3 \\
Cefotaxima & 31,7 & 27,0 & 31,4 \\
Gentamicina & 57,8 & 51,4 & 56,8 \\
Cotrimoxazol & 55,3 & 54,3 & 50,6 \\
\hline Los datos están expresados en porcentaje de cepas susceptibles.
\end{tabular}

Tabla 10. Susceptibilidad in vitro de Enterobacter cloacae aisladas en el año 2012 en población adulta

\begin{tabular}{lccc|} 
Antimicrobiano & $\begin{array}{c}\text { Hospitalizados } \\
\text { (N: } \mathbf{1 . 4 3 0}) \\
\text { \% }\end{array}$ & $\begin{array}{c}\text { UPC } \\
\text { (N: 439) } \\
\%\end{array}$ & $\begin{array}{c}\text { No UPC } \\
\text { (N: 769) } \\
\%\end{array}$ \\
Ertapenem & 82,6 & 79,3 & 81,4 \\
Imipenem & 98,6 & 96,8 & 99,4 \\
Meropenem & 98,3 & 96,3 & 99,4 \\
Amikacina & 86,3 & 86,5 & 85,7 \\
Ciprofloxacina & 72,1 & 76,3 & 70,1 \\
Piperacilina/tazobactam & 68,7 & 61,0 & 72,2 \\
Cefotaxima & 49,7 & 46,7 & 53,5 \\
Cotrimoxazol & 70,2 & 76,7 & 65,3 \\
Gentamicina & 68,8 & 58,7 & 64,2 \\
Cefepime & 81,5 & 84,4 & 78,8 \\
\hline Los datos están expresados en porcentaje de cepas susceptibles. \\
\hline
\end{tabular}

Tabla 12. Susceptibilidad in vitro de Pseudomonas aeruginosa aisladas en el año 2012 en población pediátrica

\begin{tabular}{lcc|} 
Antimicrobiano & $\begin{array}{c}\text { Hospitalizados } \\
\text { (N: } \mathbf{2 8 0}) \\
\text { \% }\end{array}$ & $\begin{array}{c}\text { UPC } \\
\text { (N: 154) }\end{array}$ \\
Gentamicina & 83,3 & $\%$ \\
Amikacina & 91,0 & 87,5 \\
Ciprofloxacina & 88,2 & 92,1 \\
Ceftazidima & 79,7 & 88,2 \\
Cefepime & 88,9 & 75,0 \\
Imipenem & 72,9 & 86,8 \\
Meropenem & 78,4 & 67,5 \\
\hline Los datos están expresados en porcentaje de cepas susceptibles. & 74,3 \\
\hline
\end{tabular}

Tabla 9. Susceptibilidad in vitro de Klebsiella pneumoniae aisladas en el año 2012 en población pediátrica

\begin{tabular}{|lcc|}
\hline & $\begin{array}{c}\text { Hospitalizados } \\
\text { (N: 396) } \\
\text { \% }\end{array}$ & $\begin{array}{c}\text { UPC } \\
\text { (N: 167) } \\
\%\end{array}$ \\
Ertapenem & 91,9 & 93 \\
Imipenem & 97,3 & 100,0 \\
Meropenem & 96,8 & 100,0 \\
Amikacina & 95,2 & 95,8 \\
Ciprofloxacina & 75,5 & 86,2 \\
Piperacilina/tazobactam & 79,4 & 83,8 \\
Cefotaxima & 62,6 & 71,9 \\
Gentamicina & 93,5 & 81,8 \\
Cotrimoxazol & 69,2 & 40,0 \\
\hline Los datos están expresados en porcentaje de cepas susceptibles. \\
\hline
\end{tabular}

Tabla 11. Susceptibilidad in vitro de Pseudomonas aeruginosa aisladas en el año 2012 en población adulta

\begin{tabular}{lccc} 
Antimicrobiano & $\begin{array}{c}\text { Hospitalizados } \\
\text { (N: } \mathbf{4 . 7 8 7 )} \\
\%\end{array}$ & $\begin{array}{c}\text { UPC } \\
\text { (N: } \mathbf{1 . 9 4 4 )}\end{array}$ & $\begin{array}{c}\text { No UPC } \\
\text { (N: } \mathbf{2 . 2 9 2})\end{array}$ \\
Gentamicina & 69,1 & 67,7 & 68,9 \\
Amikacina & 81,8 & 80,2 & 81,5 \\
Ciprofloxacina & 63,7 & 61,4 & 63,3 \\
Ceftazidima & 72,9 & 70,3 & 75,2 \\
Cefepime & 65,6 & 60,8 & 68,5 \\
Piperacilina/tazobactam & 69,2 & 61,5 & 76,6 \\
Imipenem & 65,0 & 58,0 & 73,0 \\
Meropenem & 68,3 & 62,7 & 72,5 \\
Colistín & 94,4 & 95,6 & 94,5 \\
\hline Los datos están expresados en porcentaje de cepas susceptibles.
\end{tabular}

Tabla 13. Susceptibilidad in vitro de Acinetobacter baumannii aisladas en el año 2012 en población adulta

\begin{tabular}{|c|c|c|c|}
\hline Antimicrobiano & $\begin{array}{c}\text { Hospitalizados } \\
\text { (N: } 2.055) \\
\%\end{array}$ & $\begin{array}{c}\text { UPC } \\
\text { (N: } 1.198) \\
\%\end{array}$ & $\begin{array}{c}\text { No UPC } \\
\text { (N: } 801) \\
\%\end{array}$ \\
\hline Gentamicina & 47,9 & 43,9 & 53,8 \\
\hline Amikacina & 27,6 & 25,7 & 29,9 \\
\hline Ciprofloxacina & 21,0 & 17,7 & 25,8 \\
\hline Ampicilina/sulbactam & 39,0 & 31,9 & 49,5 \\
\hline Imipenem & 30,9 & 24,0 & 40,2 \\
\hline Meropenem & 26,1 & 19,8 & 35,1 \\
\hline Colistín & 98,6 & 98,7 & 98,6 \\
\hline
\end{tabular}


Tabla 14. Susceptibilidad in vitro de Acinetobacter baumannii aislados en el año 2012 en población pediátrica

\begin{tabular}{lc} 
Antimicrobiano & $\begin{array}{c}\text { Hospitalizados (N: 58) } \\
\%\end{array}$ \\
Gentamicina & 79,6 \\
Amikacina & 94,1 \\
Ciprofloxacina & 78,9 \\
Ampicilina/sulbactam & 67,6 \\
Imipenem & 82,8 \\
Meropenem & 91,3 \\
Colistín & 100,0 \\
\hline
\end{tabular}

Los datos están expresados en porcentaje de cepas susceptibles.

de susceptibilidad in vitro a carbapenémicos, presentada por las cepas aisladas de pacientes hospitalizados, fue de $26 \%$ para meropenem y $30 \%$ para imipenem. En niños, la situación es totalmente diferente, ya que se observó mejor susceptibilidad in vitro, tanto a ampicilina/subactam $(68 \%)$ como a amikacina $(94 \%)$, carbapenémicos (83$91 \%$ ) y colistín (100\%). En general, para la mayoría de los antimicrobianos, la susceptibilidad in vitro fue inferior en unidades de pacientes críticos en comparación a las unidades de pacientes no críticos.

\section{Discusión}

El GCRB ha continuado trabajando y recolectado datos anuales procedentes de muchos establecimientos de salud; así, ha aumentado el número de hospitales participantes, obteniendo información de los años 2009, 2010, 2011 y 2012. En este reporte se presentan sólo los datos del 2012. Reportamos esta evaluación cada 2-3 años para poder evidenciar de mejor manera diferencias en susceptibilidad in vitro que ocurren a través del tiempo. Tanto para los microorganismos grampositivos como los gramnegativos se observa disminución de la susceptibilidad in vitro en relación al reporte del año 2009, a excepción de $P$. aeruginosa en que permanece estable ${ }^{1}$. Esta disminución de susceptibilidad in vitro resulta mucho más importante, por su magnitud e impacto, en las especies de $K$. pneumoniae a $\mathrm{C} 3 \mathrm{G}$ y en $A$. baumannii a carbapenémicos.

La resistencia de $S$. aureus a meticilina es relativamente similar entre los hospitales y se encuentra en alrededor de $50 \%$. La elevada susceptibilidad in vitro a cotrimoxazol y rifampicina sugiere la persistente predominancia del clon chileno ${ }^{17}$. Enterococcus faecium resistente a vancomicina es muy importante, tanto en el ámbito de pacientes críticos (UPC) como no críticos (no UPC). Se debe recordar que al solicitar la información para estos aislados se hace hincapié en que su origen sea de muestras clínicas y no de cultivos de vigilancia por lo que se podría asumir que esta susceptibilidad in vitro corresponde a verdaderas infecciones y no a la vigilancia mediante hisopado rectal que realiza la mayoría de los establecimientos. La elevada incidencia de este microorganismo debiera hacernos replantear la necesidad y costo-efectividad de mantener, sin alteraciones en 14 años, la vigilancia sistemática dentro de las políticas nacionales de prevención de infecciones ${ }^{18}$.

En enterobacterias se ha mostrado que, tanto en pacientes ambulatorios como en los pacientes hospitalizados, existe una proporción considerable de resistencia a C3G. Es difícil conseguir información exclusiva de infecciones adquiridas en la comunidad pues muchos laboratorios de establecimientos públicos, tienen como población "ambulatoria" a pacientes crónicos que han sido dados de alta en forma reciente y/o tienen morbilidades que los transforma en pacientes con altos niveles de resistencia, pero atendidos en consultorios de especialidad y subespecialidad. Por lo anterior, se espera que la situación real del paciente ambulatorio con infección comunitaria, tenga tasas de susceptibilidad in vitro mayores a las observadas en las tablas de $E$. coli uropatógenas ambulatorias.

En nuestro país, diversos estudios han demostrado la estrecha correlación que existe entre la resistencia a C3G y la presencia de BLEE, como mecanismo que la explique ${ }^{19}$. Aunque, frente a la imposibilidad de recuperar la información de los diversos software que tienen los laboratorios de la red para la gestión de microbiología, extrapolamos que la prevalencia de BLEE podría estar cercana al porcentaje de cepas no susceptibles a $\mathrm{C} 3 \mathrm{G}$, como cefotaxima o ceftriaxona. Los estudios experimentales nos indican que, actualmente en $K$. pneumoniae la BLEE predominante es CTX-M-2 y en E. coli es CTX-M-1. Sin embargo, debe destacarse que $>50 \%$ de las cepas presentan una combinación de enzimas, donde se encuentran representadas enzimas de las familias TEM y SHV ${ }^{20}$. Piperacilina/tazobactam muestra un comportamiento a cefalosporinas muy diferente dependiendo del microorganismo involucrado, con una muy buena susceptibilidad en $E$. coli y una más baja en K. pneumoniae y E. cloacae.

En Chile ya se ha descrito resistencia a carbapenémicos en enterobacterias ${ }^{21-23}$. En E. coli es infrecuente; sin embargo, en $K$. pneumoniae y E. cloacae es más importante, especialmente para ertapenem. El mecanismo de resistencia que explica este fenómeno ha sido estudiado y corresponde en gran mayoría a la hiperproducción de AmpC y/o la síntesis de BLEE, en ambos casos asociado a modificaciones en las porinas ${ }^{21}$. Si bien aún no es importante como fenómeno por su magnitud, la principal preocupación actual, tanto en hospitales públicos como privados, es la aparición de enterobacterias con resistencia a carbapenémicos mediada por la enzima $\beta$-lactamasa denominada KPC (del inglés: Klebsiella pneumoniae

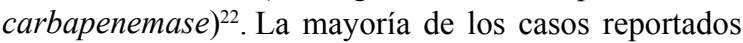
en Chile han sido explicados como casos autóctonos, con un total de 19 casos a la fecha, de acuerdo a información 
proporcionada por el Instituto de Salud Pública (datos no publicados). Esto, constituye una emergencia de salud pública en Chile. En otros países de la región, como Brasil, Argentina y Colombia, la presencia de KPC es endémica ${ }^{24}$. Se suma a esta amenaza, la reciente aparición de NDM-1 (del inglés: New Dehli metallo $\beta$-lactamase-1) en Guatemala, Colombia, Argentina, Brasil y Paraguay ${ }^{25-29}$.

En los bacilos gramnegativos no fermentadores, tanto $P$. aeruginosa como $A$. baumannii, se observa una baja susceptibilidad in vitro a carbapenémicos, con una tendencia a la disminución en la susceptibilidad, especialmente considerable, en $A$. baumannii lo que pone en una situación crítica las terapias empíricas para pacientes con infecciones por este agente, especialmente si consideramos la descripción de hasta $2 \%$ de resistencia a colistín.

Limitaciones de estos datos son los habituales reportados con la metodología utilizada. Se debe destacar que no se realizaron intervenciones a nivel local, ocupándose los estándares de calidad habituales los que incluyen además el control de calidad externo del Instituto de Salud Pública, que es universal en nuestro país. Tampoco se hizo supervisión del funcionamiento, ni de los procedimientos internos de los laboratorios. En general no hay una segregación de la información según los sitios de infección pudiendo, por ejemplo, haber diferencias entre cepas aisladas de sangre, orina o respiratorias. Esta información está sólo disponible para E. coli; por ello, no es posible relacionar la susceptibilidad in vitro de los microorganismos vigilados a ciertas infecciones de gran repercusión nosocomial como por ejemplo, neumonía asociada a ventilación mecánica-NAVM y/o infecciones del torrente saguíneoITS/catéteres venosos centrales-CVC que son de interés especialmente en unidades de pacientes críticos. Algunos antimicrobianos en particular se prueban en el laboratorio sólo en situaciones especiales, así para ellos se obtiene un número reducido de determinaciones, pudiendo generar sesgo e informar una falsa menor susceptibilidad in vitro.

La comparación de estos datos con los previamente publicados $^{1}$, no sería totalmente confiable puesto que en el actual reporte se ha incorporado un número mayor de centros de alta complejidad que en aquel año.

Es destacable el caso de tigeciclina. Por un lado, no existen puntos de corte definidos por CLSI. Por otro lado, la susceptibilidad medida por las técnicas habitualmente presentes en los laboratorios (sensidiscos, Vitek ${ }^{\circledR}$, E-test $^{\circledR}$ ) no tiene óptima concordancia con las técnicas estándares de oro en el caso de las cepas no susceptibles y por ello en el caso de hallazgo de cepas I y R se requiere volver a ensayar con métodos confirmatorios, idealmente en centros de referencia, con la técnica de microdilución en caldo, siendo el método habitual con mejor correlación Vitek ${ }^{\circledR}$ y E-test ${ }^{\circledR 30,31}$. Un estudio chileno de correlación mostró que en 724 cepas sometidas a un laboratorio de referencia, en sólo $0,1 \%$ de ellas se confirmó la resistencia (datos no publicados). Por ello, los datos de susceptibilidad in vitro para este antimicrobiano deben ser evaluados en estudio destinados a estos fines y no se muestran en este reporte.

Los datos recopilados contienen información representativa de Chile con un número considerable de aislados clínicos provenientes de un número importante de hospitales, constituyéndose por ello en información relevante para el país. En 2011 se propusieron acciones para el GCRB ${ }^{1}$. El GCRB se ha fortalecido y se ha mantenido la red de vigilancia para establecer tendencias en el tiempo, se han incorporado centros de regiones no representadas (Antofagasta, Los Ríos), se ha incorporado la medida de densidad de incidencia de los microorganismos multi-resistentes de procedencia hospitalaria y se ha podido detectar en forma precoz nuevos mecanismos de resistencia ${ }^{21}$.

Es crítico mantener información actualizada de la resistencia bacteriana en el país con el objetivo de ir evaluando y detectando resistencias emergentes, implementando medidas de contención para la prevención de la transmisión cruzada intrahospitalaria y sugiriendo terapias empíricas a los médicos clínicos. El uso de los antimicrobianos, si bien ocasiona selección de resistencia, se justifica en muchos casos por lo que queramos o no, se producirá el aumento de la resistencia. Es clave, por lo tanto, evitar la transmisión cruzada lo que permitirá a la larga, contener sólo en el paciente afectado la infección por aquellos microorganismos resistentes.

En resumen, se puede señalar que se observa una disminución de susceptibilidad in vitro de microorganismos importantes en la práctica clínica en nuestro país. Particularmente preocupa la disminución de susceptibilidad in vitro de bacilos gramnegativos no fermentadores a carbapenémicos como también de enterobacterias, dejando escasas posibilidad terapéuticas incluso en enterobacterias. Consideramos de alta importancia reportar periódicamente la resistencia bacteriana de modo de mantener a nuestra comunidad médica con información actualizada de este importante problema de salud pública. Sin embargo, creemos que el gran valor de este trabajo está en la obtención de la información local acerca de la epidemiología de la resistencia a los antimicrobianos, ya que es un fenómeno que obedece a presiones selectivas propias de cada hospital y sólo los datos locales nos pueden orientar en las terapias empíricas a implementar en cada institución en particular, como así también en las medidas de control o las intervenciones que puedan efectuarse para evitar la diseminación de cepas resistentes.

\section{Resumen}

La resistencia bacteriana es un problema de salud pública que lejos de estar controlado, aumenta en cantidad y complejidad. El Grupo Colaborativo de Resistencia, es un conjunto de profesionales que representan a 39 establecimientos de salud del país y que se ha ocupado desde 2008 de recolectar información sobre la sus- 
ceptibilidad antimicrobiana de bacterias en Chile. En este documento se presenta la susceptibilidad in vitro acumulada del año 2012, de 28 establecimientos de salud del país que representan, al menos, $36 \%$ de los egresos hospitalarios de Chile. Consideramos de la mayor relevancia reportar periódicamente la susceptibilidad bacteriana de modo de mantener a la comunidad médica actualizada para orientar las terapias empíricas y las medidas de control y prevención de la diseminación de cepas multi-resistentes.

\section{Referencias bibliográficas}

1.- Silva F, Cifuentes M, Pinto M E. Resultados de la vigilancia de susceptibilidad antimicrobiana en Chile: Consolidando una red. Rev Chilena Infectol 2011; 28 (1): 19-27.

2.- WHO. World Health Organization. The evolving threat of antimicrobial resistance: Options for action. 2012. Disponible en http://whqlibdoc. who.int/publications/2012/9789241503181_eng. pdf

3.- Woodford N, Turton J F, Livermore D M. Multiresistant Gram-negative bacteria: the role of high-risk clones in the dissemination of antibiotic resistance. FEMS Microbiol Rev 2011; 35: 736-55.

4.- Frost L S, Leplae R, Summers A O, Toussaint A. Mobile genetic elements: the agents of open source evolution. Nat Rev Microbiol 2005; 3 (9): 722-32.

5.- Amini S, Tavazoie S. Antibiotics and the post-genome revolution. Cur Opin Microbiol 2011; 14 (5): 513P 8.

6.- Davies J, Davies D. Origins and evolution of antibiotic resistance. Microbiol Mol Biol Rev 2010; 74 (3): 417-33.

7.- WHO. World Health Organization. Antimicrobial resistance: no action today, no cure tomorrow. Día Mundial de la Salud, 7 de abril 2011. Disponible en: http://www.who.int/ world-health-day/2011/en

8.- Poirel L, Labarca J, Bello H, Rioseco M L, Bernabeu S, Nordmann P. Emergence of the 16 rRNA methylase RmtG in an extended-spectrum B-lactamase producing and colistin resistant Klebsiella pneumoniae, Chile. Antimicrob Agents Chemother 2013; doi:10.1128/ AAC.02059-13

9.- Elgorriaga-Islas E, Guggiana-Nilo P, Domínguez-Yévenes M, González-Rocha G, Mella-Montecinos S, Labarca-Labarca J, et al. Prevalencia del determinante de resistencia plasmídica a quinolonas aac(6')-Ib-cr en cepas de Escherichia coli y Klebsiella pneumoniae productoras de BLEE aisladas en diez hospitales de Chile. Enferm Infecc Microbiol Clin 2012; 30: 466-8

10.- Salles M J, Zurita J, Mejía C, Villegas M V. Resistant Gram-negative infections in the outpatient setting in Latin America. Epidemiol Infect. 2013; 141 (12): 2459-72.

11.- Arias C, Murray B. Antibiotic-resistant bugs in the 21st Century-A clinical super-challenge. N Engl J Med 2009; 360: 439-43.

12.- Munita J M, Tran T, Díaz L, Panesso D, Reyes J, Murray B, et al. A liaF codon deletion abolishes daptomycin bactericidal activity against vancomycin-resistant Enterococcus faecalis. Antimicrob Agents Chemother 2013; 57 (6): 2831-3.

13.- Labarca J. Desde el aporte local al control de la resistencia bacteriana en Chile. Rev Chilena Infectol 2011; 28 (1): 12- 3.

14.- CLSI. Analysis and Presentation of Cumulative Antimicrobial Susceptibility Test Data; CLSI Document M39-A3. Wikler, MA, Clinical and Laboratory Institute. 2009.

15.- CLSI. Performance Standards for Antimicrobial Susceptibility Testing; CLSI Document M100-S22. Wayne, PA: Clinical and Laboratory Institute. 2012

16.- Norma técnica $\mathrm{N}^{\mathrm{o}} 124$ sobre programas de prevención y control de las infecciones asociadas a la atención de salud (IAAS). Exento No350, Minsal. Santiago, 24 octubre 2011. Disponible en: http://www.minsal.cl/portal/url/ item/b202490665b7804ce04001011e0148a6.pdf

17.- Rodríguez-Noriega E, Seas C. Patrón cambiante de los clones de Staphylococcus aureus resistente a meticilina en América Latina: Implicancias para la práctica clínica en la Región. Rev Chilena Infectol 2010; 27 (Supl 2): 59-69.

18.- Morgan D J, Day H R, Furuno J P, Young A, Johnson J K, Bradham D D, et al. Improving efficiency in active surveillance for methicillinresistant Staphylococcus aureus or vancomycinresistant Enterococcus at hospital admission. Infect Control Hosp Epidemiol 2010; 31 (12): 1230-5.

19.- Bello H, Trabal N, Ibáñez D, Reyes A, Domínguez M, Mella S, et al. ß-lactamasas de familias diferentes a TEM y SHV en cepas de Klebsiella pneumoniae subespecie pneumoniae aisladas en hospitales chilenos. Rev Med Chile 2005; 133: 737-9.

20.- Guggiana P, Muñoz B, Riquelme F, Hidalgo A, Elgorriaga E, González G, et al y Grupo de Estudio de Resistencia en Bacilos Gram Negativos. Identificación de betalactamasas de espectro extendido en cepas de Escherichia coli y Klebsiella pneumoniae aisladas en 10 hospitales chilenos. Rev Chilena Infectol 2011; 28 (Supl 2): S 110-3.

21.- Wozniak A, Villagra N, Undabarrena A, Gallardo N, Keller N, Moraga M, et al. Porin alterations present in non-carbapenemase producing Entero bacteriaceae with high and intermediate levels of carbapenem resistance in Chile. J Med Microbiol 2012; 61: 1270-9.

22.- Cifuentes M, García P, San Martín P, Silva F, Zúñiga J, Reyes $S$, et al. Primer caso de detección de blaKpc en Chile: desde Italia a un hospital público de Santiago. Rev Chilena Infectol 2012; 29 (2): 224-8.

23.- Gutiérrez C, Labarca J, Román J C, Sanhueza F, Moraga M, Wozniak A, et al. Vigilancia de enterobacterias productoras de carbapenemasas en cultivos rectales en un hospital universitario de Santiago, Chile. Rev Chilena Infectol 2013; 30 (1): 103-6.

24.- Castanheira M, Costello A J, Deshpande L M, Jones R N. Expansion of clonal complex 258 KPC-2-producing Klebsiella pneumoniae in Latin American hospitals: Report of the SENTRY Antimicrobial Surveillance Program. Antimicrob Agents Chemother 2012; 56 (3): 1668.

25.- Pasteran F, Albornoz E, Faccone D, Gómez S, Valenzuela C, Morales M, et al. Emergence of NDM-1-producing Klebsiella pneumoniae in Guatemala. J Antimicrob Chemother 2012; 67 (7): 1795-7

26.- INEI-ANLIS “Dr. Carlos G. Malbrán”. Emergencia de carbapenemasa tipo NDM-1 en Argentina. http://antimicrobianos.com.ar/ ATB/wp-content/uploads/2013/07/Alertaepidemiologica-NDM-Arg-v11.pdf

27.- Escobar J A, Olarte N, Castro B, Valderrama I, Garzón M, Martínez L, et al. Outbreak of NDM-1 producing Klebsiella pneumoniae in a Neonatal Unit in Colombia. Antimicrob Agents Chemother 2013; 57 (4): 1957-60.

28.- Carvalho-Assef A P, Pereira P S, Albano R, Beriao G, Chagas T, Timm L, et al. Isolation of NDM-producing Providencia rettgeri in Brazil. J Antimicrob Chemother 2013; 68 (12): 2956-7.

29.- Dirección General de vigilancia de la salud. Primer Hallazgo de Metalobetalactamasa N Delhi en Paraguay. Noviembre 2012. Disponible en: http://www.vigisalud.gov.py/attachments/ Alerta\%2006-20MetalobetalactamasaNew\%20Delhi\%20(NDM).pdf

30.- García P, Porte L, Curcio D. Estudio de susceptibilidad a tigeciclina: Influencia del agar Mueller-Hinton en el método de difusión en agar y validación del método E-test ${ }^{\circledR}$ para Acinetobacter baumannii. Rev Chilena Infectol 2009; 26 (supp1): 13- 6.

31.- García P, Cifuentes M, Juliet C, Fernández A, San Martín M, Porte L, et al. Validación de la determinación de susceptibilidad a tigeciclina con método automatizado Vitek ${ }^{\circledR}$. PosterTrabajo No 139. XXIX Congreso Chileno de Infectología. Pucón 2012. Disponible en: (http:// congreso.sochinf.cl/diplomas/2012/abstracts/ PP46.pdf?ml=5\&mlt=system \& $\mathrm{tmpl}=$ compone nt). 